\title{
Rail disruption: passenger focused recovery
}

\author{
N. Boland ${ }^{1}$, I. Evans ${ }^{2}$, C. Mears ${ }^{3}$, T. Niven ${ }^{3}$, M. Pattison ${ }^{2}$, \\ M. Wallace ${ }^{3} \&$ H. Waterer ${ }^{1}$ \\ ${ }^{1}$ The University of Newcastle, Australia \\ ${ }^{2}$ Constraint Technologies International, Australia \\ ${ }^{3}$ Monash University, Australia
}

\begin{abstract}
In a suburban passenger railway network, a delay of a single train is likely to affect not only the passengers aboard or waiting for that train, but those on subsequent trains as well. These knock-on effects are caused by the delayed train blocking sections of track and lead to congestion and slower boarding rate on overcrowded trains. When a delay has occurred, the delayed trains and other nearby trains can be re-scheduled to minimise the detrimental effect of the delay. This paper shows how to re-schedule to minimize negative impact on passengers.

A simple double track train network with a single delay is considered. The model takes into account the travel times of passengers, boarding times at stations which are lengthened when the train is crowded, and the ability of trains to bypass stations.
\end{abstract}

Keywords: passenger delay, rail, disruption, recovery.

\section{Introduction}

Disruption in rail networks can impose a substantial cost to the operator. The Melbourne passenger rail network is a radial network with morning trains essentially picking up passengers to deliver them to the hub (Flinders Street station). In this peak period the trains are running quite frequently and a common assumption to make in such a situation is that passengers arrive randomly with uniform distribution.

For trains arriving late to the final destination, one penalty incurred by the operator is measured by passenger-weighted minutes [1]. This is calculated by taking a fixed cost (roughly AU\$0.31 as at 2012 for the Melbourne network) for 
each passenger for every minute that the train is late arriving at the final destination and having a fixed expected number of passengers aboard the train. This method is designed to improve upon the method of measuring pure lateness (which penalizes a given train by the number of minutes it is late), by weighting trains in terms of the number of passengers that usually alight that train at the final station. In the case of a disruption, these penalties encourage the operator to minimize the lateness of the trains with preference to the heavily weighted trains. In the case of morning rush hour, each train collecting passengers traveling to the city conceivably has the same (or close to the same) weighting.

We look at this problem from a different perspective. Rather than just trying to recover to the original schedule after a disruption has occurred, or to minimize the arrival times at the final station, we study the rescheduling of trains with passengers in mind. In particular we consider the average passenger travel time over a time period with the goal of minimizing the impact of a disruption on the total passenger travel time whilst ensuring that all passengers arrive at their destination.

To do this, we study a simple setup of a double track line with each track only operating in one direction and thus trains cannot overtake. In such a scenario, a delay on one train can result in queuing. We also assume that passengers arrive at the station platforms at a constant rate (this constant can vary between differing stations) and that all passengers boarding a train alight at the final destination. We have in mind a morning peak period where most passengers do board a train to alight at its final destination.

We demonstrate that objective functions related to passenger travel time, which in the past may have been considered too complicated to solve, are indeed worth pursuing.

\section{A simple example of train delay and recovery}

We first consider a very simple problem consisting of three stations $\left\{S_{1}, S_{2}, S_{3}\right\}$ and two trains $\left\{T_{1}, T_{2}\right\}$. We make the following assumptions for this example:

(i) that each train starts at $S_{1}$, stops at $S_{2}$ to pick up passengers and finishes its journey at $S_{3}$,

(ii) no train can overtake any preceding train,

(iii) there are a constant number $\rho_{2}$ passengers arriving at $S_{2}$ per minute,

(iv) each train $T_{i}$ has unbounded capacity, and

(v) trains have a constant travel time of 17 minutes between stations $S_{1}$ and $S_{2}$ and 12 minutes between $S_{2}$ and $S_{3}$.

Consider the schedule,

$$
\begin{array}{ll}
d_{1,1}=0 & d_{2,1}=5 \\
a_{1,2}=17 & a_{2,2}=22 \\
d_{1,2}=20 & d_{2,2}=25 \\
a_{1,3}=32 & a_{2,3}=37
\end{array}
$$

where $d_{i, j}$ (respectively $a_{i, j}$ ) denotes the departure (respectively arrival) time of train $T_{i}$ at station $S_{j}$ relative to the start of the schedule period in minutes. 


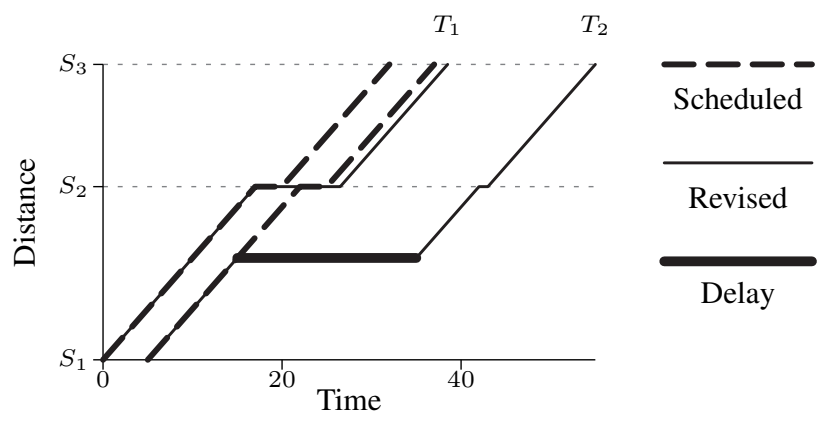

Figure 1: Simple example.

Assume that passengers begin arriving at station $S_{2}$ at time 10 or equivalently that passengers arriving before time 10 have boarded a preceding train.

Given this setup, we now introduce a delay. Suppose that $T_{2}$ is delayed between $S_{1}$ and $S_{2}$ for 20 minutes. Specifically, $T_{2}$ stops at time 15 , and resumes at time 35 to arrive at $S_{2}$ at 42 . We assume that at time 15 , the train $T_{1}$ has knowledge that $T_{2}$ will be stationary between $S_{1}$ and $S_{2}$ at time 15 and that it will resume again at 35 (see Figure 1).

Question. Can we reschedule the departure time of $T_{1}$ and $T_{2}$ from $S_{2}$ to reduce the average travel time of passengers boarding $T_{1}$ and $T_{2}$ at $S_{2}$ ?

We will assume that passengers are arriving at $S_{2}$ at a constant rate for all time $t \geq 10$. We further assume that $T_{2}$ departs $S_{2}$ at time 43 , so that it must collect passengers from $S_{2}$.

The travel time of a passenger boarding $T_{i}$, for $i \in\{1,2\}$, at $S_{2}$ is given by $x_{i, 2}+12-t$, where $x_{i, 2}$ is the rescheduled departure time of $T_{i}$ from $S_{2}$ and $t$ is the time that the passenger arrives at $S_{2}$ (we already assumed that $x_{2,2}=43$ ). Thus, the total travel time of passengers aboard $T_{1}$ and $T_{2}$ is given by

$$
\begin{aligned}
& \rho_{2} \int_{10}^{x_{1,2}} x_{1,2}+12-t d t+\rho_{2} \int_{x_{1,2}}^{43} 43+12-t d t \\
& =\rho_{2}\left(\frac{1}{2}\left(x_{1,2}-10\right)^{2}+\frac{1}{2}\left(43-x_{1,2}\right)^{2}+516\right)-120 \rho_{2},
\end{aligned}
$$

where $\rho_{2}$ is the constant rate of passenger arrival at $S_{2}$. Therefore, to minimise the total travel time, we want to find $\hat{x}_{1,2}$ for $x_{1,2}$, that minimise $\frac{1}{2}\left(x_{1,2}-10\right)^{2}+$ $\frac{1}{2}\left(43-x_{1,2}\right)^{2}+516$.

Thus $\hat{x}_{1,2}$ can be found by solving the equation

$$
\frac{d}{d \hat{x}_{1,2}}\left(\left(\hat{x}_{1,2}-10\right)^{2}+\left(43-\hat{x}_{1,2}\right)^{2}\right)=0
$$

which gives $\hat{x}_{1,2}=26.5$.

We have just shown that to minimize the total, or equivalently average, travel time of the passengers boarding $T_{1}$ and $T_{2}$ at $S_{2}$ we should reschedule the departure times of $T_{1}$ and $T_{2}$ from $S_{2}$ to 26.5 and 43 respectively. With $\rho_{2}=1$ 
this gives the total travel time of 668.25 minutes (an average of 20.25 minutes) for passengers aboard $T_{1}$ and $T_{2}$. Note that this requires $T_{1}$ to delay itself by 6.5 minutes, even though it is not physically affected by the delay of $T_{2}$. In contrast, if $T_{1}$ continued on "business as usual" and did not delay itself (so $x_{1,2}=20$ and $x_{2,2}=43$ ) then we obtain a total travel time of 710.5 (an average of 21.53 minutes).

\section{Problem formulation}

For the general case we assume that we have $m$ stations $\left\{S_{1}, S_{2}, \ldots, S_{m}\right\}$ and $n$ trains $\left\{T_{1}, T_{2}, \ldots, T_{n}\right\}$ and schedule

$$
\left\{d_{i, j} \mid 1 \leq i \leq n, 1 \leq j \leq m-1\right\} \cup\left\{a_{i, j} \mid 1 \leq i \leq n, 2 \leq j \leq m\right\},
$$

where $d_{i, j}$ refers to the scheduled departure time of $T_{i}$ from $S_{j}$ and similarly $a_{i, j}$ refers to the scheduled arrival time of $T_{i}$ at $S_{j}$.

\subsection{Assumptions}

(i) In the undisrupted schedule, each train starts at $S_{1}$, stops at each $S_{j}$ for $j=2, \ldots, m-1$ to collect passengers, and then arrives at $S_{m}$ where all passengers alight.

(ii) No train can overtake any preceding train.

(iii) There is a constant rate of passengers arriving at each station.

(iv) Passengers stop arriving at the scheduled departure time of the final train $d_{n, j}$.

(v) A more subtle requirement that we make, in rescheduling, is to forbid the departure of a given train at a given station to be sooner than its scheduled departure. This may seem unreasonable since we assume a constant rate of passenger arrival to the platforms of each station however, this does not exclude the possibility that a portion of the passengers are arriving according to the original schedule. For this reason we favor recovering to the original schedule rather than completely rescheduling.

(vi) We also allow trains to skip stations in rescheduling. This is only a problem if passengers wish to alight at stations before $S_{m}$, which we do not allow. In reality, even if some passengers do alight at intermediate stations, trains may be rescheduled to skip stations as long as the driver alerts the passengers at preceding points that this will occur.

(vii) A train that is stationary at a station cannot depart if it is not filled to capacity and there are passengers waiting to board (Section 3.4 constraint $(\mathrm{k})$ ).

\subsection{Sets and parameters}

$$
\begin{aligned}
& N=\{1,2, \ldots, n\}, N_{n}=N \backslash\{n\} \text { and } N_{1}=N \backslash\{1\} . \\
& M=\{1,2, \ldots, m\}, M_{m}=M \backslash\{m\} \text { and } M_{1}=M \backslash\{1\} . \\
& T_{i} \text { represents train } i \text { for } i \in N .
\end{aligned}
$$


$S_{j}$ represents station $j$ for $j \in M$.

$\rho_{j}$ constant rate of passengers arriving at station $S_{j}$ per time unit.

$\alpha_{j}$ minimum time for a train to move from $S_{j}$ to $S_{j+1}$, where we assume that the original schedule satisfies $a_{i, j+1}-d_{i, j} \geq \alpha_{j}$.

$c$ capacity of each train $T_{i}$.

$p_{j}$ the time that passengers begin arriving at $S_{j}$.

$h$ minimum headway between consecutive trains.

$w$ minimum dwell time at each station (if the train stops).

$u$ speed up and slow down time of the trains departing and entering stations (or simply time at the station where the train cannot collect passengers).

$i^{\prime}$ train $T_{i^{\prime}}$ is the delayed train.

$t^{\prime}$ time that $T_{i^{\prime}}$ is delayed.

$j^{\prime}$ train $T_{i^{\prime}}$ is at or approaching $S_{j^{\prime}}$ at time $t^{\prime}$.

$\delta$ duration that $T_{i^{\prime}}$ is delayed.

$\gamma$ the proportion of capacity which determines a crowded train.

$b_{1}$ the rate of passengers per time unit that can board a non-crowded train.

$b_{2}$ the rate of passengers per time unit that can board a crowded train.

\subsection{Decision variables}

Suppose that a delay has been introduced and we want to produce a new schedule that minimizes the total travel time of the passengers. For this situation we introduce the following variables.

$x_{i, j}$ the rescheduled departure time of $T_{i}$ from $S_{j}$ for $i \in N, j \in M_{m}$.

$y_{i, j}$ the rescheduled arrival time of $T_{i}$ from $S_{j}$, for $i \in N, j \in M$.

For each $i \in N$ and $j \in M_{m}$, there is a continuous interval $\sigma_{i, j}$ of time in which the people arriving at $S_{j}$ board $T_{i}$.

$\sigma_{i, j}^{l}$ the greatest lower bound of $\sigma_{i, j}$, for $i \in N, j \in M_{m}$.

$\sigma_{i, j}^{u}$ the least upper bound of $\sigma_{i, j}$, for $i \in N, j \in M_{m}$.

$c_{i, j}=\left(\sigma_{i, j}^{u}-\sigma_{i, j}^{l}\right) \rho_{j}$ the number of passengers that $T_{i}$ collects at $S_{j}$, for $i \in N$ and $j \in M_{m}$.

$l_{i, j}=\sum_{l \leq j} c_{i, l}$ the number of passengers (or load) on train $T_{i}$ at the time of departure from $S_{j}$, for $i \in N$ and $j \in M_{m}$.

Figure 2 represents a situation where $\sigma_{i, j}^{u}<x_{i, j}$. Such events may occur in reality e.g. $T_{i}$ fills to capacity at $S_{j}$ but does not immediately depart because it is traveling closely behind $T_{i-1}$, which is affected by a delay, and so chooses to wait at $S_{j}$ to maintain headway with $T_{i-1}$.

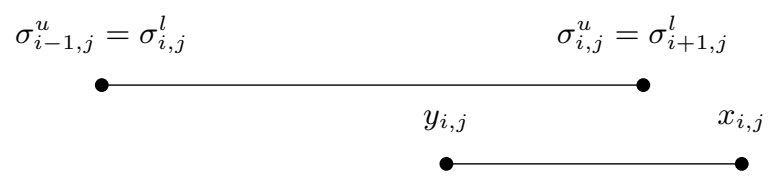

Figure 2: $\sigma_{i, j}$ interval related to train arrival and departure. 


\subsection{Constraints}

Constraints (k)-(m) involve disjunctions of linear constraints which we simply represent using the disjunction symbol $\mathrm{V}$.

(a) Fix the departure times that will not be affected by the delay.
If $d_{i, j} \leq t^{\prime}$ then $x_{i, j}=d_{i, j}$
$i \in n, j \in M$.

(b) Fix the arrival times that will not be affected by the delay.

If $a_{i, j} \leq t^{\prime}$ then $y_{i, j}=a_{i, j} \quad i \in N, j \in M$.

(c) Introduce the delay of train $T_{i^{\prime}}$.

$$
\begin{gathered}
x_{i^{\prime}, j^{\prime}} \geq t^{\prime}+\delta \text { and } \\
\text { if } t^{\prime}<a_{i^{\prime}, j^{\prime}} \text { then } y_{i^{\prime}, j^{\prime}} \geq x_{i^{\prime}, j^{\prime}-1}+\delta+\alpha_{j^{\prime}-1} .
\end{gathered}
$$

(d) No train can leave a station before it arrives.

$$
x_{i, j} \geq y_{i, j} \quad i \in N, j \in M .
$$

(e) No train departs a station before its scheduled departure time.

$$
x_{i, j} \geq d_{i, j} \quad i \in N, j \in M_{m} .
$$

(f) Each train must travel at least $\alpha_{j-1}$ time between station $S_{j-1}$ and $S_{j}$.

$$
y_{i, j+1} \geq x_{i, j}+\alpha_{j} \quad i \in N, j \in M_{m} .
$$

(g) Each train departs from $S_{1}$ before the next train (respecting the headway $h$ ).

$$
x_{i, 1}+h \leq x_{i+1,1} \quad i \in N_{1} \text {. }
$$

Each train departs a station before the next train arrives.

$$
x_{i, j}+h \leq y_{i+1, j} \quad i \in N_{n}, j \in M_{1} .
$$

(h) Each passenger at each station boards a train.

$$
\begin{array}{ll}
\sigma_{i, j}^{l} \leq \sigma_{i, j}^{u} & i \in N, j \in M_{m}, \\
\sigma_{1, j}^{l}=p_{j} & j \in M_{m}, \\
\sigma_{n, j}^{u}=d_{n, j} & j \in M_{m}, \\
\sigma_{i, j}^{u}=\sigma_{i+1, j}^{l} & i \in N_{n}, j \in M_{m} .
\end{array}
$$

(i) Capacity constraint

$$
l_{i, j} \leq c \quad i \in N, j \in M_{m} .
$$

(j) Passengers cannot board a train after it leaves.

$$
\sigma_{i, j}^{u} \leq x_{i, j} \quad i \in N, j \in M_{m}
$$

(k) Collect all passengers up until departure time subject to capacity.

$$
\begin{aligned}
& \left(\sigma_{i, j}^{l}=\sigma_{i, j}^{u}\right) \vee\left(\sigma_{i, j}^{u}=x_{i, j}\right) \vee\left(\sigma_{i, j}^{u}=d_{n, j}\right) \vee\left(c_{i, j}=c-l_{i, j-1}\right) \\
& \left(x_{i, j} \leq y_{i, j}\right) \vee\left(c=l_{i, j-1}\right) \vee\left(\sigma_{i, j}^{l}<\sigma_{i, j}^{u}\right) \\
& i \in N, j \in M_{m} \text {, } \\
& i \in N, j \in M_{m} .
\end{aligned}
$$

(1) If a train stops, then it has a minimum dwell time.

$$
\left(x_{i, j} \leq y_{i, j}\right) \vee\left(x_{i, j}-y_{i, j} \geq w+u\right) \quad i \in N, j \in M .
$$

(m) The number of passengers that board a train is dependent on the current number of passengers on the train.

$$
\begin{array}{ll}
c_{i, j} \leq b_{1}\left(x_{i, j}-y_{i, j}-u\right) & i \in N, j \in M_{m} \\
\left(l_{i, j-1} \leq \gamma c\right) \vee\left(c_{i, j} \leq b_{2}\left(x_{i, j}-y_{i, j}-u\right)\right) & i \in N, j \in M_{m} .
\end{array}
$$




\subsection{Round trip}

When returning a disrupted line to schedule, one must consider the knock-on delay of a delayed service to subsequent services which use the same rolling stock. The connecting service cannot depart its first stop until after the delayed service has reached its final stop and required activities are completed (e.g. the driver changing ends). If the connecting service is delayed, this may result in a delay to services which connect with it. To model this propagation of delay, we include round tripping, where connections between services are included. In this case the rolling stock are modeled traveling from start to end station, where all passengers alight, then express back to the start station to repeat the journey (see Figure 3 ).

\subsection{Objectives}

\subsubsection{Total travel time (TT)}

The main objective that we will be considering for this model is of average travel time of passengers (or equivalently the total travel time taken over all the passengers).

One way to view the total travel time objective function is by train. The train $T_{i}$ arrives at the final destination at time $y_{i, m}$ and at each station $S_{j}$ collects $c_{i, j}$ passengers. So there are $c_{i, j}$ passengers departing from station $S_{j}$ on train $T_{i}$ and they contribute $c_{i, j} y_{i, m}-\rho_{j} \int_{\sigma_{i, j}^{l}}^{\sigma_{i, j}^{u}} t d t=c_{i, j} y_{i, m}-\rho_{j} \frac{\left(\sigma_{i, j}^{u}\right)^{2}-\left(\sigma_{i, j}^{l}\right)^{2}}{2}$ to the total travel time. Thus the objective function can be represented as the quadratic function

$$
\begin{aligned}
& \sum_{i \in N} \sum_{j \in M_{m}}\left(c_{i, j} y_{i, m}-\rho_{j} \frac{\left(\sigma_{i, j}^{u}\right)^{2}-\left(\sigma_{i, j}^{l}\right)^{2}}{2}\right) \\
= & \sum_{i \in N} \sum_{j \in M_{m}} c_{i, j} y_{i, m}-\sum_{j \in M_{m}} \rho_{j} \frac{\left(d_{n, j}\right)^{2}-p_{j}^{2}}{2} \\
= & \sum_{i \in N} l_{i, m} y_{i, m}-\sum_{j \in M_{m}} \rho_{j} \frac{\left(d_{n, j}\right)^{2}-p_{j}^{2}}{2}
\end{aligned}
$$

Since we assume that $\rho_{j}$ is constant, we have $\mu=-\sum_{j \in M_{m}} \rho_{j} \frac{\left(d_{n, j}\right)^{2}-p_{j}^{2}}{2}$ is constant and so our objective is to minimize $\mu+\sum_{i \in N} l_{i, m} y_{i, m}$.

\subsubsection{Passenger weighted minutes (PWM)}

The passenger-weighted minutes objective function is defined (with the intention of minimizing) as

$$
\sum_{i \in N} l_{i, m}^{\prime} t_{i}
$$

where $l_{i, m}^{\prime}$ is the number of passengers on $T_{i}$ arriving at $S_{m}$ if $T_{i}$ ran to the original schedule and $t_{i}$ satisfies the following constraints 

$t_{i} \geq 0$
$i \in N$
$t_{i} \geq y_{i, m}-a_{i, m}$
$i \in N$.

Realistically, in the presence of a delay we should have $y_{i, m}-a_{i, m} \geq 0$ in which case $t_{i}$ can be replaced with $y_{i, m}-a_{i, m}$.

\subsubsection{Naive or business as usual (N)}

A "naive" or "business as usual" solution is obtained by fixing the arrival and departure times of all trains traveling before $T_{i^{\prime}}$ (the delayed train) to the original schedule and forcing all other trains affected by the delay to stop at each station and then minimizing the objective function $\sum_{i \in N, j \in M} y_{i, j}$.

\section{Implementation and results}

In this section we discuss an implementation where we assume continuous time and therefore the decision variables so far discussed are assumed to be continuous. The disjunctive constraints (constraints $(\mathrm{k}),(\mathrm{l})$ and $(\mathrm{m})$ ) will introduce binary variables, so our problem is a mixed integer problem with non-convex quadratic objective.

Using the open source solver SCIP 2.1.1 [2], which is capable of solving mixed integer problems with non-convex quadratic objective functions, we solved instances of our model with surprising success. Figure 3 shows an optimal instance of (TT) with four trains and five stations, where each train travels from $S_{1}$ to $S_{5}$ (collecting passengers along the way and then emptying at $S_{5}$ ), then express back to $S_{1}$ to repeat the journey once more. We assume that the travel from $S_{5}$ to $S_{1}$ is also first in first out without the ability to overtake. In this example $T_{3}$ is delayed for 10 minutes at time 23 minutes. At station $S_{1}, S_{2}, S_{3}$ and $S_{4}$, the numbers next

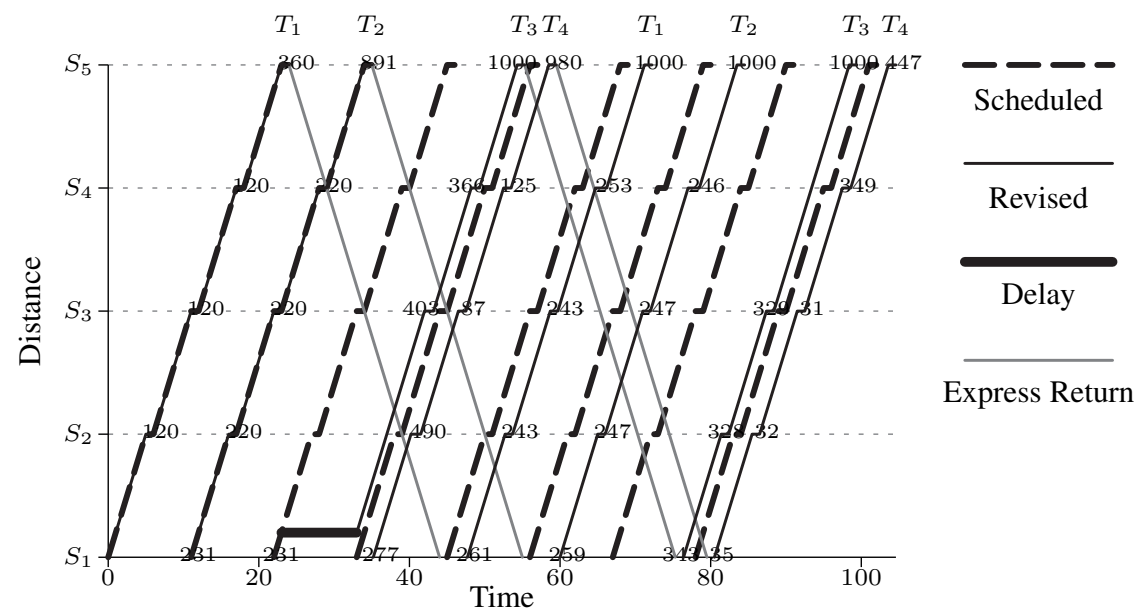

Figure 3: 10 minute delay to train 3 with round trip effects. 
to each train in Figure 3 represent the number of passengers collected at that station and the number at $S_{5}$ corresponds to total number of passengers that the train has collected or equivalently, the number of passengers alighting at $S_{5}$.

We assume that the trains have a capacity of 1000, so the boarding rate constraint (constraint $(\mathrm{m})$ from Section 3) enforces that the boarding rate slows from an original boarding rate of 600 passengers per minute, to a rate of 240 passengers per minute once the train contains 700 passengers (that is $b_{1}=600, b_{2}=240, \gamma=0.7$ and $c=1000$ ). In the example shown in Figure 3, without the boarding constraint the total travel time is 143132.6 with an average of 21.4 minutes (compared to 143936.2 with average 21.6) showing that the constraint does come into effect (if only making a negligible difference).

In Section 2 we saw that it is sometimes worthwhile for preceding trains to delay themselves in order to reduce the average travel time of passengers. In Figure 3 we see that trains $T_{1}$ and $T_{2}$ do not delay themselves on their first trip to $S_{m}$, however they do decide to delay their departure after returning to $S_{1}$ to reduce the impact of the delay on the second pass.

The (min) columns of Table 1 show the average travel time solutions that are optimal with respect to the three objective functions (TT), (PWM) and (N), applied to the example shown in Figure 3. The total number of passengers in this example is 6678 . Each result is solved to optimality within a 5 minute period on an Intel(R) Core(TM)2 Duo CPU T9300 @ 2.50GHz 2GB RAM.

Table 1: Comparison of objectives.

\begin{tabular}{c|c|cc|cc}
\hline \hline Delay Duration & $(\mathrm{TT})$ & \multicolumn{2}{|c|}{$(\mathrm{PWM})$} & \multicolumn{2}{c}{$(\mathrm{N})$} \\
& $(\mathrm{min})$ & $(\mathrm{min})$ & $(\$)$ & $(\mathrm{min})$ & $(\$)$ \\
\hline 5 & 20.38 & 22.92 & 2827.02 & 20.61 & 255.99 \\
10 & 21.55 & 23.49 & 2159.22 & 22.42 & 968.31 \\
15 & 23.91 & 25.46 & 1725.15 & 26.80 & 3216.57 \\
\hline
\end{tabular}

Interestingly, in the optimal solutions found for $(\mathrm{N})$ with 5 and 10 minute delays, the average travel time for passengers is less than that for the optimal solutions found for (PWM). Furthermore, there is no optimal solution for (PWM) that produces the same (or smaller) average travel time for passengers as that found for $(\mathrm{N})$.

A passenger hour is worth approximately AU\$10 (see [3]) and therefore an optimal solution for (TT) will provide the lowest possible cost in passenger hours. The (\$) columns of Table 1 show the extra cost incurred if the operator rescheduled with respect to (PWM) or $(\mathrm{N})$ rather than $(\mathrm{TT})$. Note that this scenario ranges over a less than a 2 hour period.

\subsection{A real world example}

We now consider a simple approximation to the Sandringham line of Melbourne's metropolitan rail network. The first station is Sandringham, the end of the spoke, 
and the fourteenth station is the hub, Flinders Street station. This scenario represents a typical morning period (in this case 80 minutes), where the third train is delayed for 10 minutes at time 25 . The number of people arriving at each station was taken from 2006 census data, that is, we took the number of people who take the train to work at each of the locations surrounding the stations and divide by schedule departure of the final train to get the passenger flow to stations.

We have 7 trains 14 stations with each train spaced 7 minutes apart. Each train has a capacity of 1300 and is considered crowded at a load of 910. A rate of 10 passengers per second can board a non-crowded train, whilst 4 passengers per second can board a crowded train. Trains must obey a headway of 90 seconds, have an acceleration and deceleration period at a station of 30 seconds and must hold doors open for a minimum of 30 seconds if collecting passengers. The passengers begin arriving at stations $S_{1}, S_{2}, \ldots, S_{14}$ at times

$$
0,0,3,6,10,11,14,18,19,23,24,27,29,0
$$

respectively. The distances (measured in minutes) between stations in order of stations are

$$
2,2,2,2,1,2,2,1,2,1,2,2,3 \text {. }
$$

The passenger arrival rates at stations $S_{1}, S_{2}, \ldots, S_{14}$ are given by

$$
4,13,11,11,5,7,14,15,12,9,14,11,6,0
$$

respectively.

Figure 4 shows a solution, with respect to the objective (TT), with a gap of 6.31 . The gap is calculated to be $\frac{T^{u}-T^{l}}{T^{l}-\mu}$, where $T^{u}$ is the bound of the total travel time, $T^{l}$ is the dual bound of the total travel time and $\mu$ is the constant defined in

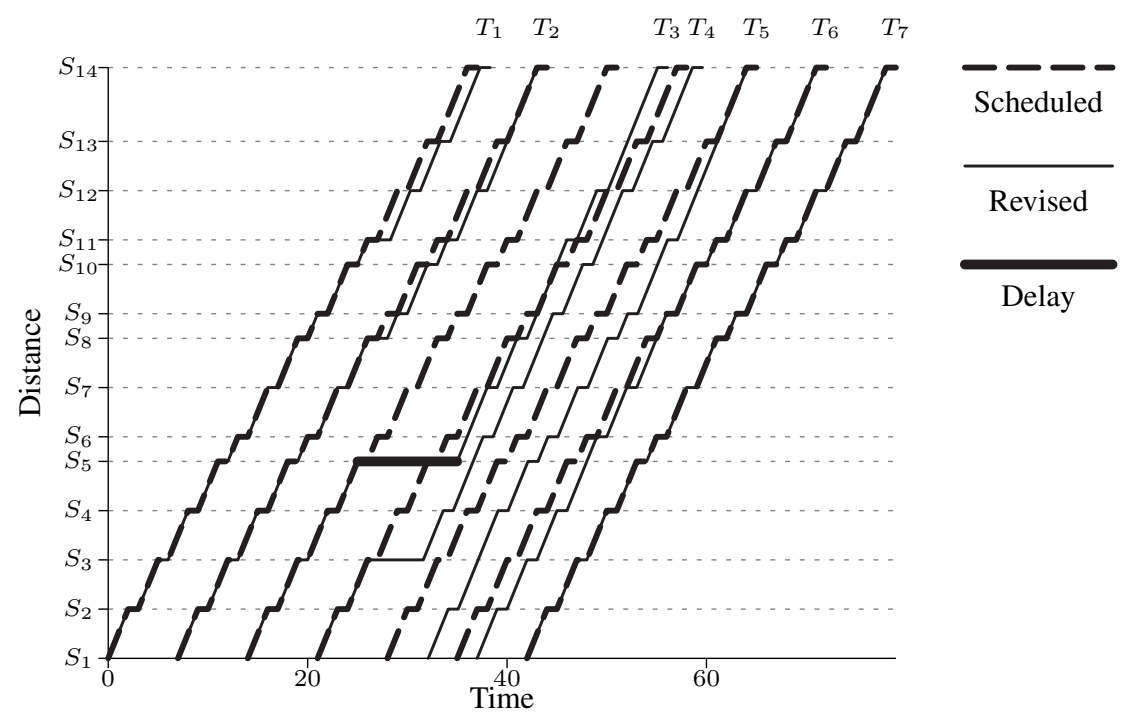

Figure 4: Minimum average travel time solution for Sandringham (TT). 
Section 3.6.1. Passengers in Figure 4 have an average travel time of 22.45 minutes whilst optimal solutions for (N) and (PWM) give average passenger travel times of 23.90 and 24.74 minutes respectively.

These results were obtained on an Intel(R) Core(TM) i7 CPU 920 @2.67GHz 12GB RAM running SCIP 2.1.1 compiled with Ipopt [4] and modelled in MiniZinc [5]. The (N) and (PWM) solutions were found in approximately 20 seconds and 1 minute of solving time respectively. The (TT) result was left running for approximately 5 hours. A smaller gap was not achieved for the (TT) solution due to RAM consumption. A solution of (TT) producing average travel times of 23.44 minutes was found in approximately 2 minutes whilst a solution of 24.18 minutes was obtained in less than 1 minute.

\section{Conclusion}

We have demonstrated that in the presence of a passenger rail network disruption, that rescheduling to minimize passenger travel time is achievable in simple rail networks. Although (PWM) appears at first glance to account for passenger travel time, we show that in fact the average travel time of passengers in a naive solution can be less than that of an optimal solution for (PWM). Thus in a rail network which penalizes the operator based on (PWM), to reduce costs, the operator would like to minimize the (PWM) objective, whilst passengers as a collective may prefer a minimal (N) solution and ultimately a minimal (TT) solution.

\section{References}

[1] Melbourne metropolitan train franchise agreement. Allens Arthur Robinson, volume 2 of 2, 2011.

[2] Achterberg, T. SCIP: solving constraint integer programs. Mathematical Programming Computation, 1(1):1-41, 2009.

[3] National guidelines for transport system management in Australia. Australian Transport Council, 2006.

[4] Wächter, A. and Biegler, L.T. On the implementation of an interior-point filter line-search algorithm for large-scale nonlinear programming. Mathematical Programming, 105(1):25-57, 2006.

[5] Nethercote, N., Stuckey, P.J., Becket, R., Brand, S., Duck, G.J., and Tack, G. Minizinc: Towards a standard CP modelling language. In Thirteenth International Conference on Principles and Practice of Constraint Programming, volume 4741 of LNCS, pages 529-543. Springer-Verlag, 2007. 